\title{
Comparando padrões de distribuição de densidade, diâmetro e abundância de espécies em áreas em processo de restauração ${ }^{1}$
}

\author{
Rafaela Pereira Naves ${ }^{2,3,4}$, Sergius Gandolfi² e Débora Cristina Rother ${ }^{2}$
}

Recebido: 30.04.2015; aceito: 2.10.2015

\begin{abstract}
Comparing patterns of density, diameter, and species abundance in areas in restoration process). In ecology, studies are mainly of observational type; there is the interest that variables in question show patterns that might be described by probability functions. The parameters of the probability distributions can be used as a signature of ecological processes occurring in the forests. In the ecology of tropical forests, few variables have the same patterns in different areas. Are these variables relevant to study areas in restoration process? In the field, we installed 90 plots in three areas (two in restoration process and one reference ecosystem). All individuals with $\mathrm{CAP} \geq 10 \mathrm{~cm}$ were measured and identified. We evaluated the empirical distributions of the variables: density, diameter at breast height, species abundance, and origin; and we fitted theoretical distributions. In the eight-year-old area, density was better described by Poisson distribution; in the 12-year-old area, negative binomial distribution; and there was no difference between these distributions in the reference ecosystem. Species abundance was better described by log-series model in the 12-year-old area, Poisson log normal in the reference ecosystem, and there was no difference between these distributions in the eight-year-old area. Weibull distribution was a good model for both areas, but parameters presented different estimates. Model fit and selection have great potential for restoration ecology.
\end{abstract}

Keywords: distribution, forest, native origin, parameters

RESUMO - (Comparando padrões de distribuição de densidade, diâmetro e abundância de espécies em áreas em processo de restauração). Estudos em ecologia são na maior parte observacionais, sendo de interesse que as variáveis em questão apresentem padrões que possam ser descritos por funções de probabilidade. Os parâmetros das distribuições de probabilidade podem ser usados como assinatura dos processos ecológicos que ocorrem nas florestas. Em ecologia de florestas tropicais, poucas variáveis apresentam os mesmos padrões em diferentes áreas. Seriam essas variáveis pertinentes para estudo de áreas em processo de restauração? Em campo, instalamos 90 parcelas em três áreas (duas em processo de restauração e um ecossistema de referência). Todos os indivíduos com CAP $\geq 10 \mathrm{~cm}$ foram medidos e identificados. Avaliamos as distribuições empíricas das variáveis densidade, diâmetro a $1,30 \mathrm{~m}$ de altura do solo, abundância de espécies e origem; e ajustamos a distribuições teóricas. Para a variável densidade, a melhor distribuição para oito anos foi Poisson; para 12 anos, binomial negativa e para ecossistema de referência não houve distinção entre essas distribuições; os melhores modelos foram os que consideraram cada área com própria estimativa dos parâmetros. Para a variável abundância de espécies, a melhor distribuição para 12 anos foi log-series; para o ecossistema de referência, foi poisson $\log$ normal; e para oito anos não houve distinção entre essas distribuições; os melhores modelos foram os que consideraram cada área com própria estimativa do parâmetro. A distribuição weibul teve bom ajuste para as três áreas para a variável DAP, mas cada área tinha suas estimativas para os parâmetros. As áreas em processo de restauração apresentaram elevada proporção de espécies de origem exótica. A abordagem de ajuste e seleção de modelos para as variáveis acima mencionadas tem grande potencial para área de ecologia da restauração.

Palavras-chave: distribuição, floresta, origem nativa, parâmetros

\section{Introdução}

Os estudos observacionais são os mais empregados em estudos ecológicos, sendo difícil replicá-los. O ambiente dificilmente é controlado e/ou manipulado.
Diante disso, percebemos barreiras para o uso da inferência estatística clássica na análise de dados provenientes de estudos desse tipo. A abordagem clássica da inferência estatística requer premissas (replicação do experimento e variâncias homogêneas,

1. Parte da Dissertação de Mestrado da primeira Autora

2. Universidade de São Paulo, Escola Superior de Agricultura "Luiz de Queiroz", Laboratório de Ecologia e Restauração Florestal, Av. Pádua Dias, 11, 13418-900 Piracicaba, SP, Brasil

3. Universidade de São Paulo, Escola Superior de Agricultura "Luiz de Queiroz", Laboratório de Métodos Quantitativos

4. Autor para correspondência email: rafaelafloresta@usp.br 
por exemplo) que muitas vezes não são possíveis obter em estudos desse tipo (Bolker 2008, Zuur et al. 2009).

Como forma alternativa a essa limitação podemos rastrear certos padrões gerados por processos no tempo e/ou espaço (Greig-Smith 1961). Esses padrões são tendências ou arranjos não aleatórios das variáveis, que podem ser considerados como assinaturas dos processos que ocorrem na organização de comunidades (Greig-Smith 1961, Lima 2013).

Em Ecologia são poucos os padrões que se mantém de uma área para outra, existindo mais exceções do que regras (Mc Gill 2007). Para a ecologia de comunidades arbóreas de florestas tropicais, podemos citar como variáveis que apresentam comportamento que se repete em diferentes áreas: o tamanho dos indivíduos arbóreos (inferido a partir dos diâmetros); a distribuição dos indivíduos entre as espécies, ou seja, a abundância das espécies e a densidade (indivíduos por área) (Magurran 2004, Nascimento et al. 2012). As primeiras duas variáveis apresentam forma conhecida como J' invertido, ou seja, existe maior concentração de observações nas menores classes (Magurran 2004, Nascimento et al. 2012, Lima et al. 2015).Os diâmetros são bem ajustados a distribuições que apresentam esta forma, como a exponencial ou distribuições que possuem certa flexibilidade como a Weibull (Meyer 1952, Nascimento et al. 2012, Lima et al. 2015) e as abundâncias de espécies pela distribuição Log-séries ou distribuição Poisson-lognormal (Fisher et al. 1943, Preston 1948). A densidade por área pode ser descrita pela distribuição de Poisson que indica ocorrência de um evento em um intervalo, desde que essa ocorrência seja aleatória. Quando existe agregação, a distribuição binomial negativa se ajusta melhor (Soares et al. 2009).

As distribuições de probabilidade possuem parâmetros, por exemplo, a distribuição normal possui o parâmetro de $\mu$ (que também é a média). A estimativa desses parâmetros pode ser usada para comparar comunidades. Supondo que a distribuição normal descreva bem determinada variável, podemos querer saber se duas comunidades submetidas a diferentes condições possuem essa variável com mesma estimativa de $\mu$ ou se cada uma apresenta seu próprio valor de $\mu$, assim esses parâmetros das distribuições de probabilidades podem ser usados como maneira indireta de caracterizar o ambiente (Diserud \& Engen 2000).

A abordagem de ajuste e seleção de modelos aparece na literatura como alternativa para a análise de dados ecológicos. Essa abordagem baseia-se na construção de modelos estocásticos (vindo da escolha de uma distribuição de probabilidade) e um componente determinístico (vindo de uma função estabelecida para os parâmetros da distribuição) (Bolker 2008). Esses modelos são, portanto, simplificações que descrevem os dados de maneira pior, ou melhor, dependendo da observação (Burnham \& Anderson 2002).

Nesse contexto utilizamos a abordagem de ajuste e seleção de modelos para a descrição de comunidades arbóreas resultantes de projetos de recuperação de áreas degradadas com restauração florestal nativa. A restauração de ambientes degradados tem longo histórico (Belloto et al. 2009), sendo a Ecologia da Restauração a ciência que estuda os processos ecológicos que ocorrem nas áreas em processo de restauração (van Andel \& Aronso 2012). A escolha de variáveis que indicarão o sucesso ou não das restaurações é parte fundamental dessa análise (Moraes et al. 2010). Em Ecologia da Restauração muitas vezes usamos as variáveis que são usadas em Ecologia de áreas naturais, relacionadas à estrutura de florestas (Rodrigues et al. 2009).

Mesmo que as áreas apresentem o mesmo padrão para as variáveis mencionadas acima, os indivíduos podem ser de espécies não oriundas da região em questão. A legislação prevê, e é de interesse para manutenção da diversidade regional, que as espécies de um plantio de restauração sejam de origem nativa (Oliveira Neto et al. 2015). No entanto, pela dificuldade de encontrar mudas ou mesmo pela dificuldade de identificação, muitas vezes são plantadas espécies oriundas de outras regiões (Mônico 2012, Oliveira Neto et al. 2015). Algumas dessas espécies se tornam competitivamente superiores às nativas, o que as caracteriza como invasoras (Richardson et al. 2000). A invasão biológica tem sido uma das causas principais de perda de diversidade em florestas tropicais (Moro et al. 2012).

O ajuste e a seleção de modelos em Ecologia da restauração são fundamentais para avaliarmos o comportamento de variáveis relacionadas à estrutura florestal e se são adequadas para testarmos diferenças entre áreas. O objetivo do presente estudo é avaliar o comportamento das variáveis: densidade (indivíduos por área); diâmetro à 1,30 m de altura do solo (DAP); abundância de espécies; e porcentagem de indivíduos de espécies de origem nativa pelo ajuste e seleção de modelos em áreas em processo de restauração e uma área de vegetação nativa presentes em uma mesma região; observar se essas variáveis podem ser descritas 
pelas mesmas distribuições de probabilidade e caso sim, se pela mesma estimativa dos parâmetros.

\section{Material e métodos}

Área de estudo - O estudo foi desenvolvido em áreas da Usina São João no município de Araras, São Paulo, Brasil. O clima é do tipo Cwa, caracterizado como tropical de altitude com chuvas no verão e seca no inverno, a altitude média é de $620 \mathrm{~m}$ e a pluviosidade anual de $1.384,5 \mathrm{~mm}$ (Centro de Pesquisas Meteorológicas e Climáticas Aplicadas à Agricultura - CEPAGRI 2011). O município encontra-se dentro do Domínio Mata Atlântica, mas com influência do Domínio Cerrado (IBGE 2004, 2007).

A paisagem onde se encontram os locais de estudo apresenta remanescentes florestais, em sua maioria, degradados e pouco conectados entre si (Isernhagen 2010). Os remanescentes da região estão inseridos em uma paisagem degradada que foi abandonada após utilização agrícola intensiva e com baixa resiliência, característica que dificulta a regeneração dos remanescentes florestais (Isernhagen 2010). A partir de estudos de adequação ambiental desenvolvidos pelo Laboratório de Ecologia e Restauração Florestal (LERF/ ESALQ/ USP), em 1998 foi dado início a um programa de restauração florestal de larga escala nas áreas de preservação permanente (APP) da Usina São João. As restaurações com plantio total foram realizadas principalmente ao redor de uma represa de abastecimento. Esses plantios foram feitos utilizando-se espécies nativas e algumas exóticas.

Coleta dos dados - Para o desenvolvimento do presente estudo foram selecionados dois reflorestamentos com espécies nativas com idades de oito e 12 anos e um remanescente florestal na região usado como ecossistema de referência.

Os plantios foram feitos utilizando-se o modelo com espaçamento $3 \times 2 \mathrm{~m}$, sendo utilizadas espécies arbustivo-arbóreas classificadas em pioneiras, secundárias e clímax, mas sem um arranjo espacial pré-definido, além de utilizar algumas espécies exóticas (Rodrigues et al. 2009). A área de oito anos é um plantio com 12,09 ha, realizado como uma faixa de cerca de $150 \mathrm{~m}$ ao redor de uma represa. Na área de 12 anos o plantio foi realizado como uma faixa de cerca de $50 \mathrm{~ms}$ ao redor da represa, totalizando 8,03 ha. O ecossistema de referência foi selecionado com base nas recomendações da SER (2004), e trata-se de um remanescente com vegetação do tipo
Floresta Estacional Semidecidual (Veloso et al. 1991), inserido no mesmo contexto das áreas em processo de restauração e submetido às mesmas pressões antrópicas, embora seja o remanesente mais bem conservado, em termos de composição e estrutura florestal da região, totalizando 96,57 ha.

Foram alocadas 30 parcelas de $10 \times 10 \mathrm{~m}$ em cada área de estudo. Dentro de cada área foi estabelecido um ponto inicial e, a partir dele, foram instaladas as parcelas distantes $10 \mathrm{~m}$ entre si e paralelas ao corpo d'água.

Para caracterizar o estrato arbustivo-arbóreo, em cada parcela foram registrados, medidos, e identificados todos os indivíduos com circunferência a 1,30 $\mathrm{m}$ de altura do solo $(\mathrm{CAP} \geq 10 \mathrm{~cm})$. Indivíduos que apresentassem bifurcação antes de $1,30 \mathrm{~m}$ foram incluídos quando a área basal de seus caules correspondesse a de um caule com CAP $\geq 10 \mathrm{~cm}$ (Silva et al. 2003). Os indivíduos foram identificados em campo quando possível ou coletados para posterior identificação no herbário ESA da Escola Superior de Agricultura "Luiz de Queiroz", por consulta em literatura especializada e a pesquisadores. As espécies foram ordenadas em famílias conforme APG III (APG III 2009).

Análise dos dados - Para avaliar os padrões das variáveis DAP, densidade, abundância de espécies de áreas em processo de restauração e uma área de vegetação nativa em uma mesma região foi utilizada a inferência baseada na verossimilhança para estimar os parâmetros e ajustar os modelos aos dados (Burnham $\&$ Anderson 2002).

A estimativa dos parâmetros foi pela máxima verossimilhança. $\mathrm{O}$ método é baseado na função de log-verossimilhança negativa:

$L\left(\Theta \mid X_{n}\right)=-\sum_{n=1}^{N}[\log [p(x)]]$

Essa função $L\left(\theta \mid X_{n}\right)$ se dá pela escolha de uma distribuição de probabilidades $p(x)$, onde fixamos os dados $\mathrm{X}_{\mathrm{n}} \mathrm{e}$ variamos os parâmetros. No valor mínimo da função temos a melhor estimativa para os parâmetros.

Uma vez escolhida a distribuição, podemos propor modelos onde os parâmetros não são constantes, pois eles variam em função de variáveis explicativas, $\theta=f(x)$.

Podemos construir modelos que descrevem os dados de maneira pior, ou melhor. É o próprio dado que vai indicar, no contexto de comparação de modelos, 
qual o mais plausível (Batista, 2009). Os modelos são comparados pelo Critério de Informação de Akaike, do inglês Akaike Information Criterion (AIC). O AIC mede a distância do modelo "verdadeiro" desconhecido ao modelo em questão, ou seja, quanto menor a distância melhor o modelo. Essa métrica é derivada da função de verossimilhança e penaliza modelos com muitos parâmetros. $\mathrm{AIC}=2 \mathrm{k}-2 \mathrm{LL}$, onde $\mathrm{k}$ é o número de parâmetros e LL é a log-verossimilhança negativa (Burnham \& Anderson 2002). O AIC é uma medida relativa que só faz sentido no contexto de comparação entre modelos, pois seu valor por si só não possui interpretação. O que nos interessa é a diferença de AIC entre dois modelos ( $\triangle \mathrm{AIC})$; desde que a diferença entre dois modelos seja menor que dois, ambos são considerados plausíveis (Burnham \& Anderson 2002).

$\mathrm{O}$ número de indivíduos arbóreos $(\mathrm{CAP} \geq 10 \mathrm{~cm})$ por parcela em cada área foi modelado através da distribuição de Poisson, que possui $\lambda$ como o único parâmetro. Esse parâmetro representa tanto a média aritmética, a esperança e a variância da distribuição (Bolker 2008). A distribuição binomial negativa também foi ajustada aos dados. Essa distribuição possui dois parâmetros, $\mu$ que é o valor esperado, e $k$ que é o parâmetro de forma que indica a agregação (Bolker 2008). Quando a média é igual a variância, a distribuição de Poisson é melhor ajustada; se variância é maior que a média, a binomial negativa tem melhor ajuste (Bolker 2008, Soares et al. 2009). Para cada área essas duas distribuições foram comparadas por meio do AIC e selecionada a distribuição que gerou o menor valor de AIC. Desde que as áreas apresentassem a mesma distribuição, foram construídos modelos onde variamos os parâmetros. Em um modelo, consideramos que as áreas apresentavam a mesma estimativa do parâmetro e, no outro modelo, cada área apresentava sua própria estimativa. Os modelos são comparados através do AIC, sendo o melhor o que apresentar o menor valor; desde que a diferença entre dois modelos seja menor que dois, ambos são considerados plausíveis (Batista, 2009).

A diversidade está relacionada à riqueza e à maneira como os indivíduos estão distribuídos entre as espécies. Existem diversas formas de expressar a diversidade de uma área e compará-la com outras (Magurran 2004). Essas diferentes abordagens variam na complexidade de metodologia e na capacidade de explicar a variação dos dados (Mc Gill et al. 2007). Escolhemos a distribuição da abundância de espécies, do inglês Species Abundance Distribution, por ser independente da composição de espécies (que pode ser diferente), e por caracterizar a estrutura das comunidades muito mais que apenas um índice de diversidade (Dornellas et al. 2009). Essas curvas, em geral, apresentam a forma de J' invertido, ou seja, a maior parte das espécies tem poucos indivíduos e poucas têm elevada abundância (Magurran 2004). Usamos as duas distribuições que tem se ajustado a diversos estudos empíricos (Hubbel 2001). A distribuição log-séries, proposta por Fisher et al. (1943), apresenta um único parâmetro $\alpha$ relacionado ao número de espécies com um indivíduo e prevê essa abundância como a de classe modal. Também ajustamos a distribuição de Poisson log-normal proposta por Preston (1948) com dois parâmetros, log-média e log-desvio. Para cada área essas duas distribuições foram comparadas pelo AIC e selecionada a distribuição que gerou o menor valor de AIC. Desde que as áreas apresentassem a mesma distribuição, foram construídos modelos onde variamos os parâmetros. Em um modelo, consideramos que as áreas apresentavam a mesma estimativa do parâmetro e, no outro modelo, cada área apresentava sua própria estimativa. Os modelos são comparados através do AIC, sendo o melhor aquele que apresentar o menor valor; desde que a diferença entre dois modelos seja menor que dois, ambos são considerados plausíveis (Batista, 2009).

A distribuição do diâmetro a $1,30 \mathrm{~m}$ de altura do solo (DAP) em florestas tropicais em geral apresenta a forma de $\mathrm{J}$ ' invertido, sendo bem descrita por distribuições que permitam essa forma. Os dados foram truncados no limite de inclusão, pois não é possível conhecer o comportamento dessa variável abaixo desse limite. Posteriormente, foi ajustada a distribuição exponencial que apresenta $\lambda$ como parâmetro e é o inverso da média, e também a distribuição Weibull, com os parâmetros de forma e escala (Bolker 2008, Lima et al. 2015). Para cada área, essas duas distribuições foram comparadas através do AIC e selecionada a distribuição que gerou o menor valor de AIC. Desde que as áreas apresentassem a mesma distribuição, foram construídos modelos onde variamos os parâmetros. Em um modelo, consideramos que as áreas apresentavam a mesma estimativa do parâmetro e, no outro modelo, cada área apresentava sua própria estimativa. Os modelos são comparados através do AIC, sendo o melhor o que apresentar o menor valor; desde que a diferença entre dois modelos seja menor que dois, ambos são considerados plausíveis (Batista 2009). 
Tendo em vista que, mesmo que as áreas em processo de restauração apresentem as variáveis densidade, abundância de espécies e DAP com comportamento semelhante ao remanescente, essas áreas podem ter dentre os indivíduos alguns que são espécies exóticas. As espécies foram classificadas quanto às suas origens (nativa ou exótica da Floresta Estacional Semidecidual do interior do Estado de São Paulo). Como espécie exótica entendemos ser aquela levada para fora de sua distribuição geográfica por transporte humano de forma intencional ou não (Richardson et al. 2000). Com isso obtivemos a informação de porcentagem de espécies nativas ocorrentes em cada parcela de cada área. A distribuição binomial é a ideal para tratar dados de proporção, possuindo um único parâmetro $p$ que varia de 0 a 1 e é relacionado à proporção de sucessos em certo número de tentativas (no nosso caso número de indivíduos de espécies nativas pelo total de indivíduos) (Bolker 2008); foram construídos modelos onde variamos esse parâmetro. Em um modelo, consideramos que as áreas apresentavam a mesma estimativa do parâmetro $e$, no outro modelo, cada área apresentava sua própria estimativa. Os modelos são comparados através do AIC, sendo o melhor aquele que apresentar o menor valor; desde que a diferença entre dois modelos seja menor que dois, ambos são considerados plausíveis (Batista, 2009).

Todas as análises foram feitas no software $\mathrm{R}$ (R Core Team 2014). As funções dpois, dnbinom, dexp, dweibull, dbinom foram utilizadas como funções de probabilidade e a função dpoilog do pacote poilog (Grotan \& Engen 2008) para função poisson log normal na construção da função de log-verossimilhança negativa, sendo utilizada a função mle2 do pacote bbmle para minimizá-la (Bolker 2014). Para ajuste das distribuições das abundâncias das espécies, utilizamos a função fitsad do pacote sads (Prado \& Miranda 2014).

\section{Resultados e Discussão}

Para a variável número de indivíduos por parcela, a distribuição binomial negativa foi a que teve melhor ajuste para a área de 12 anos, a área de oito anos teve melhor ajuste com a distribuição Poisson, enquanto para o remanescente não houve distinção entre as duas distribuições, de acordo com o AIC (tabela 1). Construímos modelos para comparar o ecossistema de referência com cada uma das áreas em processo de restauração, considerando o modelo Poisson para comparação com a área de oito anos e o binomial negativo para de 12 anos; esses modelos indicaram que o comportamento da variável número de indivíduos por parcela não foi o mesmo entre as áreas (tabela 2). Os modelos ajustados aos dados são mostrados na figura 1.

A distribuição binomial negativa já foi encontrada como a que melhor representa dados de contagem de indivíduos de comunidades biológicas com média maior que variância e ela pode estar ligada ao padrão de dispersão dos indivíduos (Bolker 2008, Soares et al. 2009) refletindo o processo natural de organização de comunidades (Hutchings 1997). As áreas em processo de restauração do presente estudo são recentes, o número de indivíduos por parcela pode ainda ser resultado das condições do plantio e manutenção.

$\mathrm{O}$ valor esperado de densidade (indivíduos ha ${ }^{-1}$ ) foi de 1.100 ind ha $^{-1}$ para a área de oito anos, 1.486 ind ha $^{-1}$ para a área de 12 anos e 1.813 ind ha $^{-1}$ para o ecossistema de referência. Esses valores estão dentre os observados em outros estudos, tanto para áreas em processo de restauração (Souza 2000, Pulitano et al. 2004), quanto para áreas naturais (Ivanauskas et al. 1999, Durigan et al. 2000). A variável densidade de fato é uma variável de fácil obtenção em estudos ecológicos e a partir dela podemos inferir a ocupação no espaço, mas dificilmente apenas por meio dela podemos inferir sobre os processos ecológicos que ocorrem como mortalidade, recrutamento, competição por recursos entre outros. Nesse caso, avaliações temporais (repetidas ao longo do tempo) são importantes para inferir tais processos.

Para a variável abundância de espécies, o ecossistema de referência foi mais bem representado pela distribuição Poisson log-normal, enquanto a área de 12 anos pela log-séries, para área de oito anos não houve distinção entre essas duas distribuições. Diante disso as comparações foram feitas entre a área de oito anos e a de 12, considerando o modelos logséries, e entre a área de oito anos e o ecossistema de referência, considerando o modelo Poisson log-normal (tabela 3). Esses modelos indicaram que as áreas não apresentaram as mesmas estimativas dos parâmetros. A figura 2 traz as distribuições empíricas e as teóricas ajustadas para o modelo escolhido.

Como acontece em quase todas as comunidades, as do presente estudo também apresentaram o padrão em J' invertido para abundância das espécies (Magurran 2004). A distribuição log-série é geralmente encontrada quando existe a maior agregação de espécies na primeira classe (1), sendo essa a modal, 
Tabela 1. Ajuste e seleção de distribuições para as variáveis: densidade (indivíduos por parcela), abundância de espécies e DAP $(\mathrm{cm})$ para duas áreas em processo de restauração e um ecossistema de referencia. AIC: Akaike Information Criterion; $d$ AIC: diferença entre esses valores; $D F$ : graus de liberdade do modelo. O melhor modelo está representado em negrito.

Table 1. Model fit and selection for the variables: density (individuals per plot), species abundance and diameter at breast height $(\mathrm{cm})$ for three sites (eight years, 12 years, and reference ecosystem). AIC: Akaike Information Criterion; dAIC: difference between these values; $d f$ : degree of freedom. The best model is in bold.

\begin{tabular}{lccccc}
\hline Variável & Área & Distribuição & AIC & dAIC & df \\
\hline & oito anos & Poisson & 150,0 & 0 & 1 \\
& oito anos & Binomial negativa & 152,2 & 2,2 & 2 \\
Indivíduos.parcelas $^{-1}$ & 12 anos & Binomial negativa & 195,9 & 0 & 2 \\
& 12 anos & Poisson & 219,0 & 23,0 & 1 \\
& Ecossistema Referência & Poisson & 184,5 & 0 & 1 \\
& Ecossistema Referência & Binomial negativa & 184,9 & 0,4 & 2 \\
& oito anos & Log-series & 328,1 & 0 & 1 \\
& oito anos & Poisson Log-normal & 328,5 & 0,4 & 2 \\
Abundância de espécies & 12 anos & Log-series & 297,1 & 0 & 1 \\
& 12 anos & Poisson Log-normal & 302,2 & 5,1 & 2 \\
& Ecossistema Referência & Poisson Log-normal & 315,4 & 0 & 2 \\
& Ecossistema Referencia & Log-series & 329,7 & 14,2 & 1 \\
& oito anos & Weibull & $2.323,4$ & 0 & 2 \\
dap (cm) & oito anos & Exponencial & $2.323,9$ & 0,6 & 1 \\
& 12 anos & Weibull & $2.686,3$ & 0 & 2 \\
& 12 anos & Exponencial & $2.703,2$ & 16,8 & 1 \\
& Ecossistema Referência & Weibull & $2.837,0$ & 0 & 2 \\
& Ecossistema Referencia & Exponencial & $3.020,1$ & 183,2 & 1 \\
\hline
\end{tabular}

Oito anos

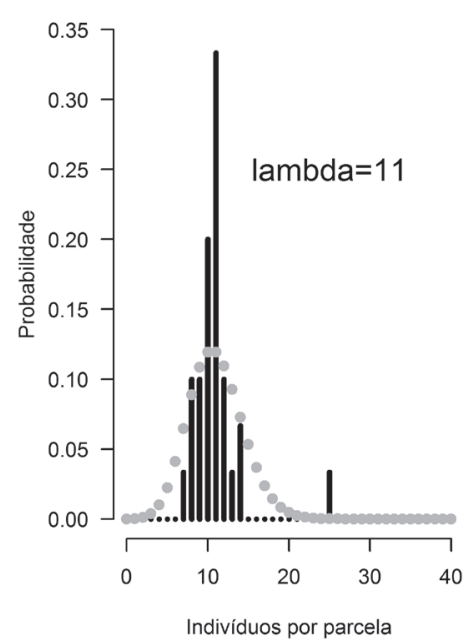

12 anos

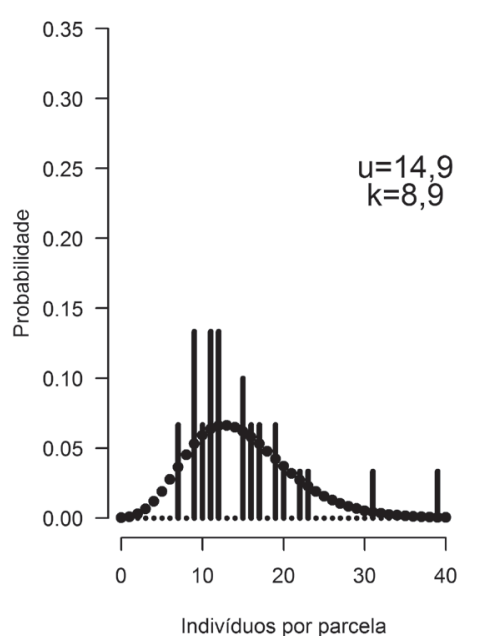

Ecossistema de Referência

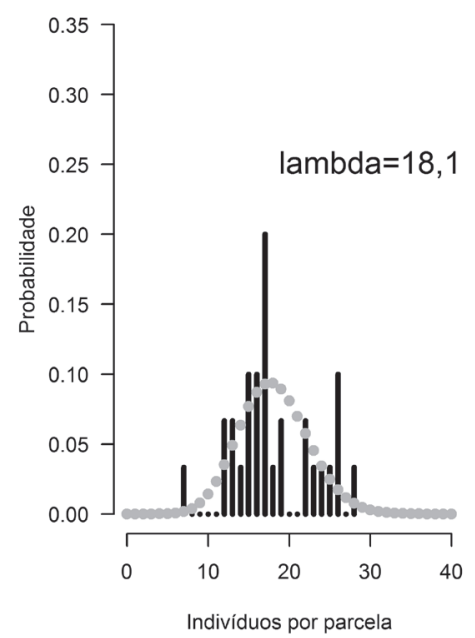

Figura 1. Distribuição empírica das frequências de indivíduos por parcela em três áreas (oito anos, 12 anos e ecossistema de referência). Em cinza, o modelo teórico da distribuição de Poisson ajustado aos dados onde cada área tem seu próprio $\lambda$ (lambda); em preto, o modelo teórico da distribuição binomial negativa.

Figure 1. Empirical distribution of individual frequency per plot in three sites (eight years, 12 years, and reference ecosystem). Fitted Poisson is shown in grey while fitted negative binomial is shown in black points. Each site presents its own parameters. 
Tabela 2. Modelos criados para descrever número de indivíduos por parcela para duas áreas em processo de restauração e um ecossistema de referência. A comparação entre área de oito anos e ecossistema de referência foi feita pelo modelo Poisson e entre área de 12 anos e ecossistema de referência com modelos binomial negativo. DF: graus de liberdade; AIC: Akaike Information Criterion; dAIC: diferença entre esses valores. Em negrito, o melhor modelo.

Table 2. Models to describe density in three sites (eight years, 12 years, and reference ecosystem). Models were built with Poisson and Negative Binomial distribution. AIC: Akaike Information Criterion; dAIC: difference between these values; $d f$ : degree of freedom. The best model is in bold.

\begin{tabular}{|c|c|c|c|c|c|}
\hline Áreas & Modelo & Descrição & $d f$ & AIC & $d \mathrm{AIC}$ \\
\hline Ecossistema de referência & M1 & $\mathrm{X} \sim$ Poisson $(\mathrm{x} \mid \lambda=($ área $))$ & 2 & 334,5 & 0 \\
\hline e oito anos & M0 & $\mathrm{X} \sim \operatorname{Poisson}(\mathrm{x} \mid \lambda=($ constante $))$ & 1 & 385,4 & 50,9 \\
\hline Ecossistema de referência & M1 & $\mathrm{X} \sim \mathrm{BN}(\mathrm{x} \mid \mu=($ área $), \mathrm{k}=($ constante $))$ & 2 & 354,1 & 0 \\
\hline e 12 anos & M0 & $\mathrm{X} \sim \mathrm{BN}(\mathrm{x} \mid \mu=($ constante $), \mathrm{k}=($ constante $))$ & 1 & 361,4 & 7,3 \\
\hline
\end{tabular}

Tabela 3. Modelos criados para descrever abundância das espécies para duas áreas em processo de restauração e um ecossistema de referência. A comparação entre área de oito anos e ecossistema de referência foi feita através e modelo Poisson log normal e entre área de 12 anos e oito anos com modelos Log-series. DF: são os graus de liberdade; AIC: Akaike Information Criterion; $d$ AIC: diferença entre esses valores. Em negrito, o melhor modelo.

Table 3. Models to describe species abundance to three sites (eight years, 12 years and Reference Ecosystem). Models were built with Poisson log normal and log series distribuction. AIC: Akaike Information Criterion; $d$ AIC: difference between these values; $d f$ : degree of freedom. The best model is in bold.

\begin{tabular}{|c|c|c|c|c|c|}
\hline Áreas & Modelo & Descrição & $\mathrm{df}$ & $\mathrm{AIC}$ & dAIC \\
\hline Ecossistema de referência & M1 & $\mathrm{X} \sim$ Poisson-log normal (x | $\mu=($ área $), \sigma=($ área $)$ ) & 4 & 643,9 & 0 \\
\hline e oito anos & M0 & $\mathrm{X} \sim$ Poisson-log normal $(\mathrm{x} \mid \mu=($ constante $), \sigma=($ constante $))$ & 2 & 648,7 & 4,8 \\
\hline 12 anos & M1 & $\mathrm{X} \sim \log -\operatorname{series}(\mathrm{x} \mid \alpha=$ (área) $)$ & 2 & 354,1 & 0 \\
\hline e oito anos & M0 & $\mathrm{X} \sim \log$-series $(\mathrm{x} \mid \alpha=($ constante $))$ & 1 & 361,4 & 7,3 \\
\hline
\end{tabular}

Oito anos

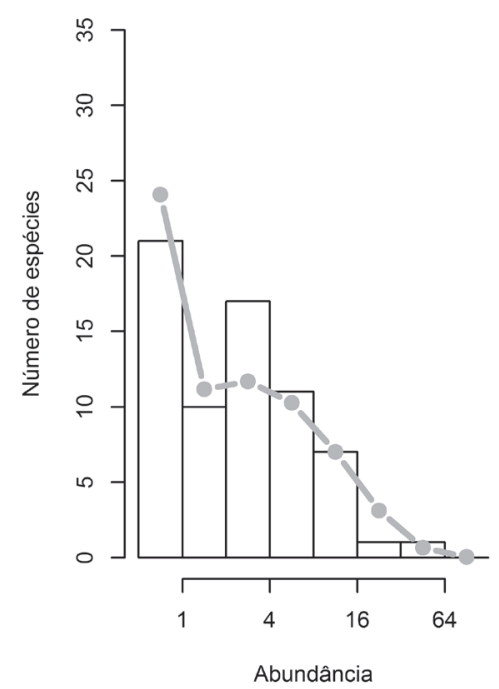

12 anos

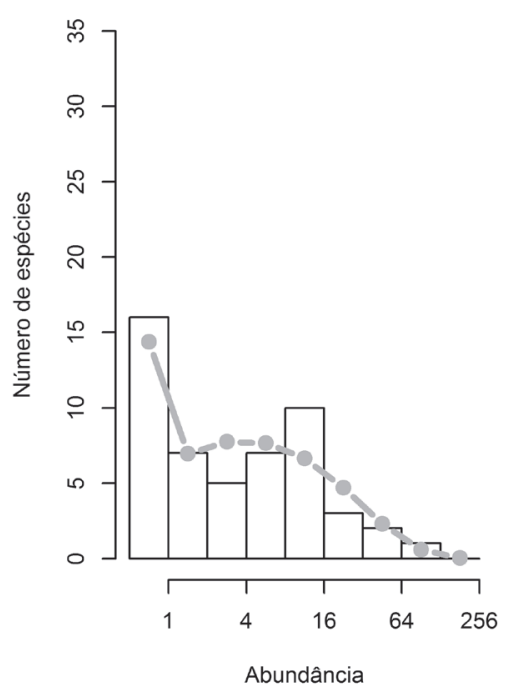

Ecossistema de Referência

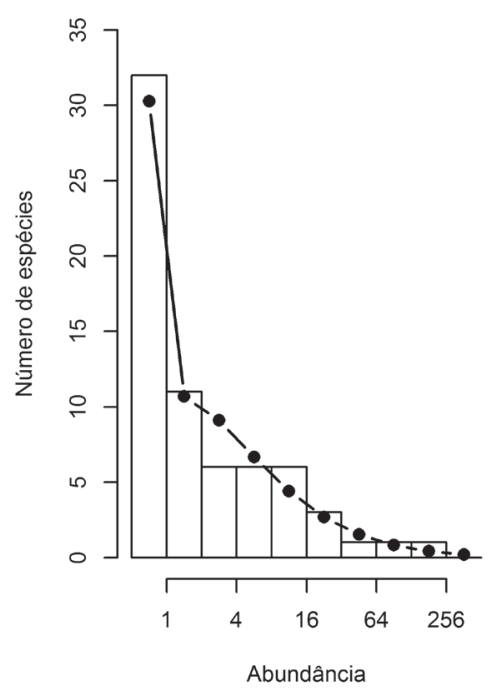

Figura 2. Distribuição empírica das abundâncias das espécies em três áreas (oito anos, 12 anos e ecossistema de referência). Em cinza, o modelo ajustado da distribuição Log-series e em preto, modelo ajustado da distribuição de Poisson Log-normal.

Figure 2. Empirical distribution of species abundance in three sites (eight years, 12 years, and reference ecosystem). Fitted Log-series is shown in grey while fitted Poisson Log-normal is shown in black points. Each site presents its own parameters. 
o único parâmetro $\alpha$ é relacionado ao número de espécies com apenas um indivíduo (Fisher et al. 1943). Já a distribuição de Poisson log-normal (encontrada para o ecossistema de referência) é reportada para comunidades onde isso não ocorre (Preston 1948). Segundo Preston (1948), a classe modal ser a de abundância 1 também pode ser devido ao fato da amostra ser pequena. Essas distribuições geralmente estão relacionadas à partição de recursos entre as espécies e à dinâmica da comunidade (Diserud \& Engen 2000, Mandai 2010) e distúrbios podem alterar a sua forma (Dornellas et al.2009). A premissa de que padrões de abundância de espécies podem expressar a estrutura de uma comunidade ecológica é o conceito central que suporta a construção dos modelos de distribuição de abundância de espécies (Tokeshi1993). Entretanto, o ajuste de dados reais a esses modelos não significa que os padrões observados possam ser explicados por suas premissas, já que estes modelos não incorporam todos os fatores que atuam sobre a comunidade ecológica (por ex., estocasticidade e interações positivas e negativas). Embora a distribuição de probabilidade e as estimativas dos parâmetros para representar a abundância das espécies nas áreas em processo de restauração tenham sido diferentes do ecossistema de referência, ela indicou que os plantios avaliados têm padrão de abundância de espécies esperado para comunidades biológicas (J' invertido) naturais.

A distribuiçãoWeibull foi escolhida para representar o DAP $(\mathrm{cm})$ para as três áreas, embora para a área de oito anos não houve distinção entre essa distribuição e a exponencial negativa (tabela 1). Embora a família de distribuição escolhida tenha sido a mesma, o melhor modelo foi o que previu cada área tendo seus próprios parâmetros de forma e escala (tabela 3). A figura 3 traz as densidades empíricas e o ajuste do modelo 4.

A distribuição Weibull é bem flexível e se ajustou a outros estudos empíricos de diâmetro em Florestas Tropicas (Lima et al. 2015). Esse tipo de forma ( $\mathrm{J}$ 'invertido) está relacionado à dinâmica de florestas. Diversos fatores atuam na dinâmica da estrutura horizontal de florestas formadas por indivíduos de diferentes espécies e diferentes idades (mortalidade de indivíduos, crescimento, incremento, variações das condições climáticas, distúrbios, entre outros), causando heterogeneidade no tempo e no espaço (Nascimento et al. 2012). A competição por recursos e a mortalidade é maior nas menores classes diamétricas, assim a maior parte dos indivíduos representada nas menores classes de tamanho retrata o processo de regeneração, enquanto a sua ausência ou menor densidade de indivíduos nessas classes pode ser indicativo de não estar ocorrendo recrutamento (Meyer 1952, Nascimento et al. 2012). As áreas em processo de restauração já apresentam essa forma para essa variável, mas ainda assim pode ser resultado também das condições do plantio e não somente de processos ecológicos.

Para áreas naturais, a forma de J' invertido da distribuição do DAP é resultante da dinâmica da floresta; para áreas em processo de restauração, principalmente as recentes, temos diferentes espécies plantadas em um mesmo momento crescendo com diferentes taxas e o processo de regeneração natural iniciando. Com essa variável obtemos a ocupação dos indivíduos (distribuição dos diâmetros) na área em restauração, mas sua interpretação não deve ser a mesma que em áreas naturais.

As áreas em processo de restauração apresentaram muitos indivíduos de espécies não nativas das Florestas Estacionais Semideciduais do interior do Estado de São Paulo (figura 4).

Foram criados modelos para a proporção de indivíduos de origem nativa por parcela utilizando-se a distribuição binomial (tabela 5). O melhor modelo foi o que previu que cada área apresenta o seu próprio parâmetro $\left(p_{8 \text { anos }}=0,51, p_{12 \text { anos }}=0,44\right.$, $\left.p_{\text {Ecossistema de Referência }}=0,94\right)$, esse parâmetro $p$ indica a proporção de indivíduos pertencentes a espécies de origem nativa por parcela, sendo que as áreas em processo de restauração apresentaram valores menores.

Outros estudos já reportaram a presença de espécies exóticas em plantios, principalmente nos plantios mais antigos (Preiskorn 2011, Mônico 2012). Por fazerem parte da estrutura florestal das áreas em restauração, inserir as espécies exóticas nas análises é de extrema importância. Por esse motivo não retiramos os indivíduos de espécies exóticas das outras análises (densidade, DAP, abundância). Do ponto de vista da conservação, mesmo tendo contribuição "negativa", essas espécies fazem parte da comunidade e influenciam sua estrutura, pela competição por recursos e espaço (Moro et al. 2012). Essa é uma variável que merece especial atenção, e deve ser mais bem estudada, pois pode comprometer o objetivo da restauração ecológica de restaurar os processos ecológicos do ecossistema.

Analisamos neste trabalho o comportamento de variáveis que são comumente mensuradas em 
Tabela 4. Modelos criados para descrever DAP $(\mathrm{cm})$ para duas áreas em processo de restauração e um ecossistema de referência, onde a variável DAP $(\mathrm{X})$ pode ser descrita pela distribuição Weibull, que possui parâmetros ( $\alpha$ : forma, $\beta$ : escala), que por sua vez variam de acordo com outras variávies. $d f$ são os graus de liberdade; AIC: AkaikeInformationCriterion; $d$ AIC: diferença entre esses valores. Em negrito, o melhor modelo.

Table 4. Models to describe diameter at breast height $(\mathrm{cm})$ in three sites (eight years, 12 years, and reference ecosystem). The variable was described by a Weibull distribution, where its parameters ( $\alpha$ : shape, $\beta$ : scale) could vary. AIC: Akaike Information Criterion; $d$ AIC: difference between these values; $d f$ : degree of freedom. The best model is in bold.

\begin{tabular}{|c|c|c|c|c|}
\hline Modelo & Descrição & df & AIC & dAIC \\
\hline M4 & $\mathrm{X} \sim$ Weibull (x $\mid \alpha=\mathrm{f}($ área), $\beta=\mathrm{f}$ (área)) & 6 & $7.846,6$ & 0 \\
\hline M1 & $\mathrm{X} \sim$ Weibull( $\mathrm{x} \mid \alpha=\mathrm{f}$ (plantio ou não), $\beta=\mathrm{f}($ plantio ou não) $)$ & 4 & $7.881,6$ & 35,0 \\
\hline M2 & $\mathrm{X} \sim$ Weibull( $\mathrm{x} \mid \alpha=$ (constante),$\beta=\mathrm{f}$ (área) $)$ & 4 & $7.887,4$ & 40,8 \\
\hline M3 & $\mathrm{X} \sim$ Weibull( $\mathrm{x} \mid \alpha=\mathrm{f}$ (área), $\beta$ (constante)) & 4 & $7.946,0$ & 99,3 \\
\hline M0 & $\mathrm{X} \sim$ Weibull $(\mathrm{x} \mid \alpha$ (constante), $\beta$ (constante) $)$ & 2 & $7.965,7$ & 119,1 \\
\hline
\end{tabular}

Tabela 5. Modelos criados para descrever proporção de espécies nativas por parcela para duas áreas em processo de restauração e um ecossistema de referência, onde a variável $(\mathrm{X})$ pode ser descrita pela distribuição binomial, que possui o parâmetro $p$, que por sua vez varia de acordo com outras variávies. DF: graus de liberdade; AIC: Akaike Information Criterion; $d$ AIC: diferença entre esses valores. Em negrito, o melhor modelo.

Table 5. Models to describe the proportion of native individuals per plot in three sites (eight years, 12 years, and reference ecosystem). The variable can be described by a binomial distribution and the parameter $p$ could vary. AIC: Akaike Information Criterion; $d$ AIC: difference between these values; $d f$ : degree of freedom. The best model is in bold.

\begin{tabular}{ccccr}
\hline Modelo & Descrição & $\mathrm{df}$ & AIC & dAIC \\
\hline M2 & $\mathrm{X} \sim \operatorname{Binomial}(\mathrm{x} \mid \mathrm{p}=\mathrm{f}($ área) $)$ & 3 & 471,6 & 0 \\
M1 & $\mathrm{X} \sim \operatorname{Binomial}(\mathrm{x} \mid \mathrm{p}=\mathrm{f}$ (plantio ou não) $)$ & 2 & 473,6 & 2,0 \\
M0 & $\mathrm{X} \sim \operatorname{Binomial}(\mathrm{x} \mid \mathrm{p}=($ constante) $)$ & 1 & 843,9 & 372,3 \\
\hline
\end{tabular}

Oito anos

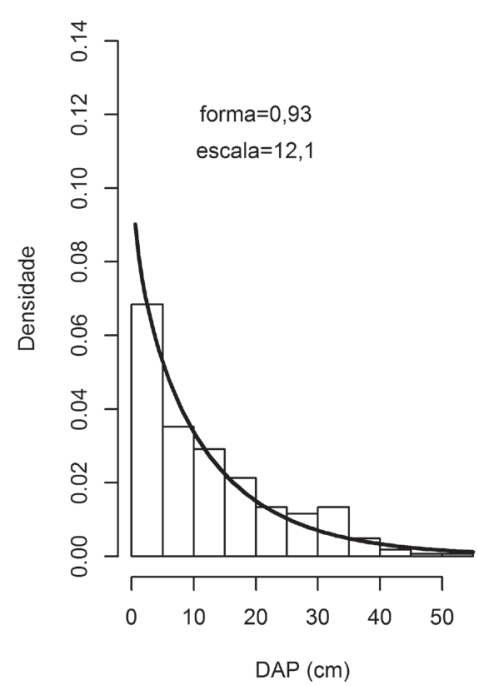

12 anos

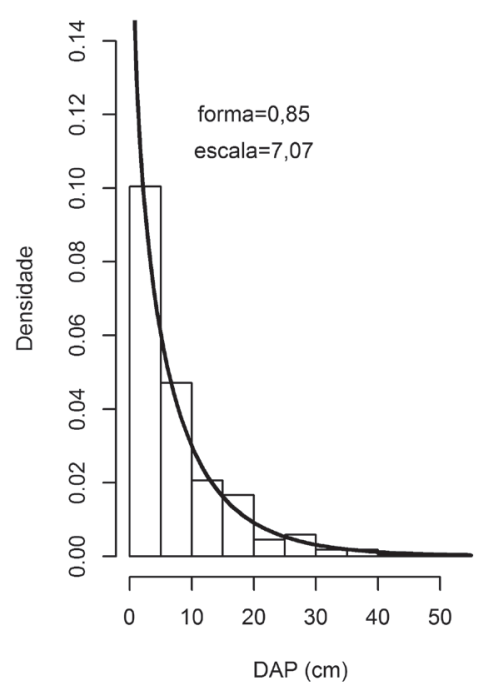

Ecossistema de Referência

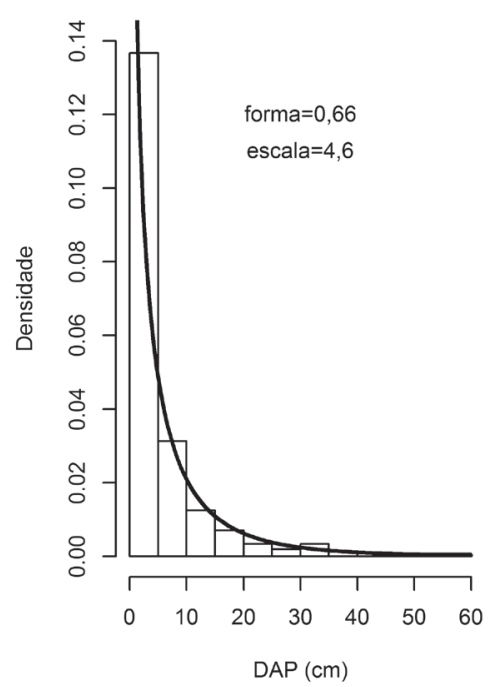

Figura 3. Distribuição empírica do DAP $(\mathrm{cm})$ em três áreas (oito anos, 12 anos e ecossistema de referência) e em preto o modelo teórico da distribuição Weibull onde cada área tem seus próprios parâmetros de forma e escala.

Figure 3. Empirical distribution of diameter at breast height in three sites (eight years, 12 years, and reference ecosystem). Fitted Weibull is shown in black line. Each site presents its own parameters. 


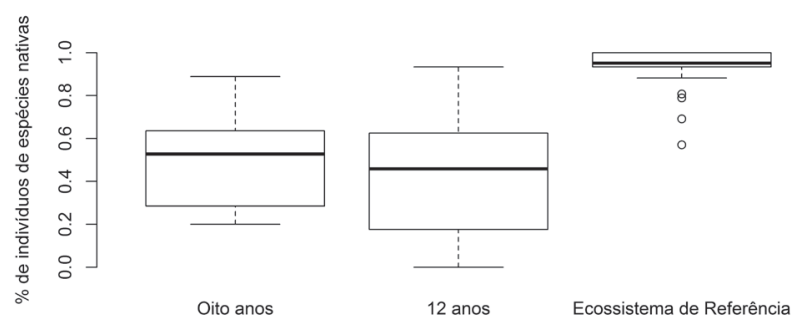

Figura 4. Proporção de indivíduos de espécies nativas por parcela em áreas em processo de restauração (oito e 12 anos) e ecossistema de referência. São apresentados os limites inferior e superior, a mediana e o $3^{\circ}$ e $4^{\circ}$ quartis.

Figure 4. Proportion of individuals of native species per plot in three sites (eight years, 12 years, and reference ecosystem). The upper and lower limits, the median, and the 3rd and 4rd quantiles are presented.

trabalhos de ecologia de florestas tropicas: densidade (indivíduos por área), tamanho (DAP), abundância das espécies e presença de espécies exóticas. Para variável densidade, a melhor distribuição para oito anos foi Poisson, 12 anos binomial negativa e ecossistema de referência não houve distinção entre essas distribuições; os melhores modelos foram os que consideraram cada área com própria estimativa dos parâmetros. Para a variável abundância de espécies, a melhor distribuição para 12 anos foi log-séries, ecossistema de referência foi poisson log-normal e oito anos não houve distinção entre essas distribuições; os melhores modelos foram os que consideraram cada área com própria estimativa do parâmetro. A distribuição Weibul teve bom ajuste para as três áreas para a variável DAP, mas cada área tinha suas estimativas para os parâmetros. As áreas em processo de restauração apresentaram elevada proporção de espécies de origem exótica.

Para áreas em processo de restauração, como as do presente estudo, existe ainda o efeito das condições como as quais foram realizadas o plantio, sendo difícil distinguir quais são os efeitos de processos ecológicos, quais os de práticas silviculturais e qual a interação entre eles. Essas variáveis devem ter interpretação diferente daquela para áreas naturais.

Apesar disso, o uso de tais variáveis é extremamente útil para descrever a estrutura e a diversidade das áreas em restauração, especialmente das áreas de maior idade nas quais os processos resultantes das relações espécie $\times$ espécie e espécie $\times$ ambiente nas escalas de indivíduo. A abordagem de ajuste e seleção de modelos mostrou-se extremamente útil na análise dessas variáveis e comparação entre áreas, visto que essa abordagem é mais flexível.

\section{Agradecimentos}

Os autores agradecem ao Conselho Nacional de Desenvolvimento Científico e Tecnológico, pela bolsa de Mestrado concedida à primeira Autora (processo 130245/2011-9) e pelo apoio financeiro do projeto temático (processo 561897/2010-7); à Usina São João, à Fazenda Campo Alto, por disponibilizar as áreas; aos colegas do Laboratório de Ecologia e Restauração Florestal (LERF), ESALQ, USP, que ajudaram na coleta dos dados e identificação das plantas.

\section{Literatura citada}

APG III. 2009. An update of the Angiosperm Phylogeny Group classification for the orders and families of flowering plants: APG III. Botanical Journal of the Linnean Society 161: 105-121.

Batista, J.L.F. 2009. Verossimilhança e Máxima Verossimilhança. Apostila de estudo. Instituição: ESALQ/USP Piracicaba.

Belloto, A, Gandolfi, S. \& Rodrigues, R.R. 2009. Fase 1: Restauração fundamentada no plantio de árvores, sem critérios ecológicos para a escolha e combinação das espécies. In: R.R. Rodrigues, P.H.S. Brancalion, I. Isernhagen, I. Pacto para a restauração da Mata Atlântica: referencial dos conceitos e ações de restauração florestal. 3 ed. ESALQ, LERF/Instituto BioAtlântica, São Paulo. pp. 11-14.

Bolker, R.B. \& R Development Core Team. 2014. bbmle: Tools for general maximumlikelihoodestimation. $\mathrm{R}$ package version 1.0.17. Disponível em http://CRAN.Rproject.org/package=bbmle (acesso em 11-X-2015).

Bolker, B. 2008. Ecological Models and Data in R Princeton. Princeton University Press, Princeton.

Burnham, K.P. \& Anderson, D.R. 2002. Model selection and multi-model inference: a practical information theoretic approach, 2 ed. Springer, New York.

CEPAGRI - Centro De Pesquisas Meteorológicas e Climáticas Aplicadas à Agricultura. 2011. Clima dos municípios paulistas. Disponível em http://www.cpa. unicamp.br/outras-informacoes/clima_muni_038.html (acesso em 15-X-2012).

Diserud, O.H. \& Engen, S. 2000. A general and dynamic species abundance model, embracing the lognormal and the gamma models. The American Naturalist 155: 497-511.

Dornelas, M., Moonen, A. C., Magurran, A. E., \& Bàrberi, P. 2009. Species abundance distributions reveal environmental heterogeneity in modified landscapes. Journal of Applied Ecology. 46: 666-672. 
Durigan, G., Franco, G.A.D.C., Saito, M. \& Baitello, J.B. 2000. Estrutura e diversidade do componente arbóreo da floresta na Estação Ecológica dos Caetetus, Gália, SP. Revista Brasileira de Botânica 23: 371-383.

Fisher, R.A., Corbet, A.S., \& Williams, C.B. 1943. The relation between the number of the species and the number of individuals in a random sample from animal population. Journal of Animal Ecology 12: 42-58.

Greig-Smith, P. 1961. Data on Pattern within Plant Communities: I. The Analysis of Pattern. The Journal of Ecology 149: 695-702.

Grotan, V. \& Engen, S. 2008. poilog: Poisson lognormal and bivariate Poisson lognormal distribution. R package version 0.4

Hubbel, S.P. 2001. The Unied Neutral Theory os Biodiversity and Biogeography. University Press, Princeton.

Hutchings, M.J. 1997. The structure of plant populations. In: M.J. Crawley (ed.). Plant ecology. Blackwell Science, Oxford, pp. 97-136.

IBGE - Instituto Brasileiro de Geografia e Estatística. 2004. Mapa de Biomas do Brasil: primeira aproximação. Rio de Janeiro. Disponível em http://www.ibge.gov.br/ home/presidencia/noticias/noticia_visualiza.php?id_ noticia=169 (acesso em 11-X-2015).

IBGE - Instituto Brasileiro De Geografia e Estatística. 2007. Perfil dos municípios brasileiros. Disponível em http://www.ibge.gov.br/cidadesat/topwindow.htm?1 (acesso em 11-X-2015).

Isesrnhagen, I. 2010. Uso de semeadura direta de espécies arbóreas nativas para restauração florestal em áreas agrícolas. Tese de Doutorado, Universidade de São Paulo, Piracicaba.

Ivanauskas, N.M., Rodrigues, R.R. \& Nave, A.G. 1999. Fitossociologia de um trecho de Floresta Estacional Semidecidual em Itatinga, São Paulo, Brasil. Scientia Forestalis 56: 83-99.

Lima, R.A.F. 2013. Ajuste e seleção de modelos na descrição de comunidades arbóreas: estrutura, diversidade e padrões espaciais. Tese de Doutorado, Universidade de São Paulo, São Paulo.

Lima, R.A.F., Batista, J.L.F. \& Prado, P.I. 2015. Modeling tree diameter distributions in natural forests: An evaluation of 10 statistical models. Forest Science. 61: 320-327.

Magurran, A.E. 2004. Measuring biological diversity. Blackwell, Oxford, pp. 285-286.

Mandai, C.Y. 2010. Aplicação de modelos teóricos de distribuição de abundância das espécies na avaliação de efeitos de fragmentação sobre as comunidades de aves da Mata Atlântica. Dissertação de Mestrado, Universidade de São Paulo, São Paulo.
McGill, B.J., Etienne, R.S., Gray, J.S., Alonso, D., Anderson, M.J., Benecha, H.K., Dornelas, M., Enquist, B.J., Green, J.L., He, F., Hurlbert, A.H., Magurran, A.E., Marquet, P.A., Maurer, B.A., Ostling, A., Soykan, C.U., Ugland, K.I. \& White, E.P. 2007. Species abundance distributions: moving beyond single prediction theories to integration within an ecological framework. Ecology Letters 10: 995-1015.

Meyer, H.A. 1952. Structure, growth, and drain in balanced uneven-aged forests. Journal of forestry 50: 85-92.

Mônico, A.C. 2012. Transferência de bancos de sementes superficiais como estratégia de enriquecimento de uma floresta em processo de restauração. Dissertação de Mestrado, Universidade de São Paulo, Piracicaba.

Moraes, L.F.D., Campello, E.F.C. \& Franco, A.A. 2010. Restauração florestal: do diagnóstico de degradação ao uso de indicadores ecológicos para o monitoramento das ações. Oecologia Australis 14: 437-451.

Moro, M.F., Souza, V.C., Oliveira-Filho, A.T., Queiroz, L.P., Fraga, C.N., Rodal, M.J.N., Araújo, FS. \& Martins, F.R. 2012. Alienígenas na sala: o que fazer com espécies exóticas em trabalhos de taxonomia, florística e fitossociologia. Acta Botanica Brasilica 26: 991-999.

Nascimento, R.G.M., Machado, S.A., Figueiredo Filho, A., \& Higuchi, N. 2012. Modelo de projeção por classe diamétrica para florestas nativas: enfoque na função probabilística de Weibull. Pesquisa Florestal Brasileira 32: 209 .

Oliveira Neto, N.E., Fonseca, C.R., \& Carvalho, F.A. 2015. O problema das espécies arbóreas exóticas comercializadas nos viveiros florestais: Estudo de caso no município de Juiz de Fora (MG). Revista de Biologia Neotropical 11: 28-46.

Prado, P.I, \& Miranda, M.D. 2014. sads: Maximum Likelihood Models for Species Abundance Distributions. $\mathrm{R}$ package version 0.1.10.

Preiskorn, G.M. 2011. Composição florística, estrutura e quantificação de carbono em florestas restauradas com idades diferentes. Dissertação de Mestrado, Universidade de São Paulo, Piracicaba.

Preston, F.W. 1948. The commonness and rarity of species. Ecology 29: 254-283.

Pulitano, F.M., Durigan, G.\& Dias, L.E. 2004. A mata ciliar da Fazenda Cananéia: estrutura e composição florística em dois setores com idades diferentes. In: O. Vilas Boas, G. Durigan (orgs.). Pesquisas em conservação e recuperação ambiental no Oeste Paulista: resultados da cooperação Brasil/Japão. Páginas \& Letras, São Paulo pp. 419-445.

R Development Core Team. 2014. R: A Language and Environment for Statistical Computing. 
Richardson, D.M., Pysek, P., Rejmánek, M., Barbour, M.G., Panetta, F.D. \& West, C.J. 2000. Naturalization and invasion of alien plants: concepts and definitions. Diversity and Distributions, Oxford 6: 93-107.

Rodrigues, R.R., Brancalion, P.H.S. \& Isernhagen, I. (orgs.). 2009. Pacto para a restauração da Mata Atlântica: referencial dos conceitos e ações de restauração florestal. 3 ed. ESALQ, LERF/Instituto Bio Atlântica, São Paulo.

SER Society For Ecological Restoration International Science \& Policy Working Group. 2004. The SER international primer on ecological restoration. 2 ed Tucson: Society for Ecological Restoration International. Disponível em http://www.ser.org (acesso em 11-X-2015).

Silva, J.M.C. \& Casteleti, C.H.M. 2003. Status of the biodiversity of the Atlantic Forest en Brazil. In: C. Galindo-Leal \& I.G. Câmara (eds.). The Atlantic Forest of South America: biodiversity status, threats, and outlook. CABS/Island Press, Washington. pp. 43-59.
Soares, C.P.B., Rodello, C.M., Souza, A.L., Garcia, H., Leite, V.P.S., \& Silva, G.F. 2009. Comparação entre procedimentos de amostragem para espécies florestais raras e padrão de distribuição espacial agregado. Revista Árvore 33: 545-553.

Souza, F.M. 2000. Estrutura e dinâmica do estrato arbóreo e da regeneração natural em áreas restauradas. Dissertação de Mestrado, Universidade de São Paulo, Piracicaba.

Tokeshi, M. 1993. Species Abundance Patterns and Community. Advances in ecological research 24: 111.

Van Andel, J. \& Aronso, J. (eds). 2012. Restoration Ecology: the new frontier. John Wiley \& Sons, Oxford.

Veloso, H.P., Rangel Filho, A.L.R. \& Lima, J.C.A. 1991. Classificação da vegetação Brasileira adaptada a um sistema universal. FIBGE, Rio de Janeiro.

Zuur, A., Ieno, E.N., Walker, N., Saveliev, A.A., \& Smith, G.M. 2009. Mixed effects models and extensions in ecology with R. Springer Science \& Business Media, New York. 